\title{
RESTRICTING THE STEINBERG CHARACTER IN FINITE SYMPLECTIC GROUPS
}

\author{
JIANBEI AN AND GERHARD HISS
}

\begin{abstract}
We determine the restriction of the Steinberg character of a finite symplectic group of odd characteristic to its maximal parabolic subgroup stabilizing a line. We relate this restriction to the tensor product of a Weil character with the Steinberg character. As an application we prove that Donovan's conjecture has a positive answer for unipotent $\ell$-blocks of the six-dimensional symplectic groups of odd characteristic when $\ell>3$.
\end{abstract}

\section{Introduction}

Parabolic subgroups are "large" subgroups of finite groups of Lie type. Thus knowledge about the representations of a parabolic subgroup should yield information about the representations of the group itself. Since in general the irreducible representations of a parabolic subgroup $P$ cannot be classified, one usually restricts attention to representations of $P$ having its unipotent radical in their kernel.

In classical groups there are distinguished parabolic subgroups, whose Levi subgroups are classical groups of the same type, and whose ordinary irreducible representations can be classified. This is the case, for example, for a finite symplectic group $G=\operatorname{Sp}_{2 m}(q)$ and its maximal parabolic subgroup $P$ fixing a line in the natural representation. It makes sense to ask for the restrictions of the irreducible characters of $G$ to $P$.

In this paper we determine the restriction of the Steinberg character of $G$ to $P$ if $q$ is odd. The parametrization of the irreducible characters of $P$ is rather different in the two cases of odd and even $q$. In the latter case, $G \cong S O_{2 m+1}(q)$, and this parametrization resembles the one for the groups $S O_{2 m+1}(q)$ with odd $q$. Hence the case of even $q$ is best dealt with in the framework of orthogonal groups.

Assume now that $q$ is odd and let $U$ denote the unipotent radical of $P$ and $L$ its standard Levi subgroup. Then $L$ is a direct product of a cyclic group of order $q-1$ and of $L^{\prime}=\operatorname{Sp}_{2 m-2}(q)$. The irreducible characters of $P$ with $U$ in their kernels are in bijection with the irreducible characters of $L$. The irreducible characters of $P$ whose kernel is the centre of $U$, are parametrized by the irreducible characters of the parabolic subgroup $P_{2 m-2}$ of $L^{\prime}$ analogous to $P$. Finally, there is a fourto-one correspondence between the remaining irreducible characters of $P$ and the irreducible characters of $L^{\prime}$.

The restriction of the Steinberg character of $G$ to $P$ contains exactly one constituent of the first type, namely the Steinberg character of $L$. The constituents of the second type can be computed by restricting the Steinberg character of $L^{\prime}$ to $P_{2 m-2}$. Characters of the third type correspond to the constituents of the product of the Weil character with the Steinberg character of $L^{\prime}$. 
We explicitly compute all the constituents in the cases $G=\operatorname{Sp}_{4}(q)$ and $\operatorname{Sp}_{6}(q)$. It turns out that these restrictions are multiplicity free. We use this result to show that if $\ell$ is a prime bigger than 3 , all unipotent $\ell$-blocks of a given defect occurring in the groups $\operatorname{Sp}_{6}(q)$ for odd prime powers $q$ not divisible by $\ell$, lie in finitely many Morita equivalence classes. For this purpose we use an approximation to the decomposition matrix of these groups. Such an approximation is not available for $\ell=2$ or for groups $\operatorname{Sp}_{2 m}(q)$ when $m$ is bigger than 3 . We could perhaps extend the above result to the case $\ell=3$, but only at the cost of rather tedious technicalities.

We expect that the ideas used in this paper can be extended to other classical groups to yield qualitative information about decomposition numbers of unipotent characters.

\section{The GRoups AND SOME SUbGroups}

2.1. The groups. We begin by fixing some notation which will be used throughout our paper. Let $q$ be a power of a prime $p$, and let $\mathbb{F}_{q}$ denote the finite field with $q$ elements. Also, $m$ is a positive integer and $n:=2 m$. We consider the finite symplectic group $\operatorname{Sp}_{n}(q)$ over the field $\mathbb{F}_{q}$. For the computations we are going to perform it is convenient to work with a specific natural matrix representation of this group which we now introduce.

Matrices are usually, but not always, denoted by boldface lower case letters, the transposed of a matrix $\mathbf{a}$ is written as $\mathbf{a}^{t}$. We write $I_{m}$ for the identity matrix of degree $m$, and $J_{m}$ for the $(m \times m)$-matrix with ones along the anti-diagonal and zeroes otherwise. Finally, let $\tilde{J}_{n}$ be the matrix

$$
\tilde{J}_{n}:=\left[\begin{array}{cc}
0 & J_{m} \\
-J_{m} & 0
\end{array}\right] .
$$

With these conventions we define

$$
G:=\operatorname{Sp}_{n}(q):=\left\{\mathbf{x} \in \operatorname{GL}_{n}(q) \mid \mathbf{x}^{t} \tilde{J}_{n} \mathbf{x}=\tilde{J}_{n}\right\} .
$$

We sometimes refer to $n$ as the degree of $G$. The Weyl group of $G$ is of type $C_{m}$.

2.2. The parabolic subgroup. We let $P$ be the maximal parabolic subgroup of $G$ fixing a line (in the natural $G$-vector space $\mathbb{F}_{q}^{n}$ ). Then the Levi subgroup $L$ of $P$ is (up to a cyclic direct factor) a symplectic group of degree $n-2$ (we adopt the convention that $\operatorname{Sp}_{0}(q)=\{1\}$ ). Occasionally we consider the analogous parabolic subgroup of $L$, and so on, in a recursive fashion. We therefore indicate the degree of the ambient symplectic group by a subscript.

We now describe $P$ through its Levi decomposition. First of all, for $\mathbf{v} \in \mathbb{F}_{q}^{n-2}$ and $z \in \mathbb{F}_{q}$ we put

$$
\mathbf{u}_{n}(\mathbf{v}, z):=\left[\begin{array}{ccc}
1 & \mathbf{v}^{t} \tilde{J}_{n-2} & z \\
0 & I_{n-2} & \mathbf{v} \\
0 & 0 & 1
\end{array}\right] .
$$

Then, we let $U_{n} \leq G$ be the subgroup

$$
U_{n}:=\left\{\mathbf{u}_{n}(\mathbf{v}, z) \mid \mathbf{v} \in \mathbb{F}_{q}^{n-2}, z \in \mathbb{F}_{q}\right\} .
$$

Next, for $\mathbf{x} \in \operatorname{Sp}_{n-2}(q)$ and $a \in \mathbb{F}_{q}^{*}$, we write

$$
\mathbf{s}_{n}(\mathbf{x}, a):=\left[\begin{array}{ccc}
a & 0 & 0 \\
0 & \mathbf{x} & 0 \\
0 & 0 & a^{-1}
\end{array}\right],
$$


and put

$$
L_{n}:=\left\{\mathbf{s}_{n}(\mathbf{x}, a) \mid \mathbf{x} \in \operatorname{Sp}_{n-2}(q), a \in \mathbb{F}_{q}^{*}\right\} .
$$

Then $P:=P_{n}:=U_{n} L_{n}$ is a maximal parabolic subgroup of $G=\operatorname{Sp}_{n}(q)$ fixing the line $\left\langle[1,0, \ldots, 0]^{t}\right\rangle$. The group $U_{n}$ is the unipotent radical of $P$ and $L_{n}$ is its Levi complement. We write $L_{n}^{\prime}$ for the subgroup of $L_{n}$ consisting of the matrices $\mathbf{s}_{n}(\mathbf{x}, a)$ of (1) with $a=1$. Then $L_{n}^{\prime} \cong \operatorname{Sp}_{n-2}(q)$. (Moreover, $L_{n}^{\prime}$ is the commutator subgroup of $L_{n}$ except if $n=4$ and $q=2,3$ or $n=6$ and $q=2$.) Also, $L_{n}=L_{n}^{\prime} \times A$, where $A=\left\{\mathbf{s}_{n}\left(I_{n-2}, a\right) \mid a \in \mathbb{F}_{q}^{*}\right\}$. We finally put $P_{n}^{\prime}:=U_{n} L_{n}^{\prime}$.

If $n=2$ or $q$ is even, then $U_{n}$ is abelian. If $n>2$ and $q$ is odd, then $U_{n}$ is a special $p$-group of structure $q^{1+(n-2)}$. In this case, the center $Z\left(U_{n}\right)$ of $U_{n}$ is isomorphic to the additive group of $\mathbb{F}_{q}$ and consists of the matrices $\mathbf{u}_{n}(0, z), z \in \mathbb{F}_{q}$, the central quotient $U_{n} / Z\left(U_{n}\right)$ is isomorphic to the natural vector space $\mathbb{F}_{q}^{n-2}$, and the action of $L_{n}^{\prime}$ on $U_{n} / Z\left(U_{n}\right)$ by conjugation is equivalent to the natural action of $\operatorname{Sp}_{n-2}(q)$ on $\mathbb{F}_{q}^{n-2}$.

2.3. The characters of $P_{n}$. Assume that $n \geq 2$, and put $P:=P_{n}, U:=U_{n}$, $L:=L_{n}, L^{\prime}:=L_{n}^{\prime}$ and $P^{\prime}:=U L^{\prime}$. The ordinary irreducible characters of $P$ can be classified into three types:

Type 1: Characters with $U$ in their kernel.

Type 2: Characters with $Z(U)$ but not $U$ in their kernel.

Type 3: Characters with $Z(U)$ not in their kernel.

Note that for $n=2$ there are no characters of Type 2 (since $Z(U)=U$ in this case). Using Clifford theory, we are now going to describe the characters of the individual types in more detail and introduce a convenient notation.

2.3.1. Characters of Type 1. These are in bijection with $\operatorname{Irr}(L)$ via inflation. We write ${ }^{1} \psi_{\sigma}$ for the character of Type 1 corresponding to $\sigma \in \operatorname{Irr}(L)$. Thus

$$
{ }^{1} \psi_{\sigma}=\operatorname{Infl}_{L}^{P}(\sigma) .
$$

We have ${ }^{1} \psi_{\sigma}(1)=\sigma(1)$.

2.3.2. Characters of Type 2. These are parametrized by $\operatorname{Irr}\left(P_{n-2}\right)$ in the following way. A character of Type 2 is a character of the quotient $P / Z(U)$ which is a semidirect product of $L$ with $U / Z(U)$. Since $L^{\prime}$ is transitive on the non-zero vectors of its natural module, there is just one $L^{\prime}$-orbit on the set of non-trivial characters of $U / Z(U)$. The irreducible characters of Type 2 thus correspond to the irreducible characters of the stabilizer in $L$ of a non-trivial irreducible character of $U / Z(U)$. Again we need a convenient choice for the purpose of computations.

Suppose that $n \geq 4$ and let $\xi$ be a nontrivial irreducible complex character of the additive group of $\mathbb{F}_{q}$. Define $\lambda \in \operatorname{Irr}(U)$ by $\lambda\left(\mathbf{u}_{n}(\mathbf{v}, z)\right):=\xi\left(v_{1}\right)$, where $\mathbf{v}=\left[v_{1}, \ldots, v_{n-2}\right]^{t} \in \mathbb{F}_{q}^{n-2}$. Let $T_{n}:=T_{P}(\lambda)$ denote the inertia subgroup of $\lambda$ in $P$. It is readily checked that $T_{n}=U_{n} \tilde{P}_{n-2}$ with $\tilde{P}_{n-2}=\tilde{U}_{n-2} \tilde{L}_{n-2}$, where the subgroups $\tilde{U}_{n-2}$ and $\tilde{L}_{n-2}$ are embedded into $L$ in the following way (missing entries in the matrices below are zeroes):

$$
\tilde{U}_{n-2}=\left\{\left[\begin{array}{l|l|l}
1 & & \\
\hline & \mathbf{u}_{n-2}(\mathbf{v}, z)^{t} & \\
\hline & & 1
\end{array}\right] \mid \mathbf{v} \in \mathbb{F}_{q}^{n-4}, z \in \mathbb{F}_{q}\right\},
$$


and

$$
\tilde{L}_{n-2}=\left\{\left[\begin{array}{c|ccc|c}
a^{-1} & & & & \\
\hline & a & 0 & 0 & \\
& 0 & \mathbf{x} & 0 & \\
& 0 & 0 & a^{-1} & \\
\hline & & & & a
\end{array}\right] \mid \mathbf{x} \in \operatorname{Sp}_{n-4}(q), a \in \mathbb{F}_{q}^{*}\right\} .
$$

Clearly, $\tilde{P}_{n-2} \cong P_{n-2}$, and thus there is a bijection between $\operatorname{Irr}\left(\tilde{P}_{n-2}\right)$ and $\operatorname{Irr}\left(P_{n-2}\right)$. We construct a particular such bijection to describe the characters of Type 2. First, we put $P_{n-2}^{*}:=\left\{\mathbf{s}_{n}\left(\left(\mathbf{x}^{-1}\right)^{t}, 1\right) \mid \mathbf{x} \in P_{n-2}\right\}$. Then $P_{n-2} \cong P_{n-2}^{*}$, and we write $\mu \mapsto \mu^{*}$ for the corresponding bijection of irreducible characters. We then have $P_{n-2}^{*} \times A=\tilde{P}_{n-2} \times A$ (recall that $A$ consists of the matrices $\mathbf{s}_{n}\left(I_{n-2}, a\right)$ for $\left.a \in \mathbb{F}_{q}^{*}\right)$. Hence the map $\operatorname{Irr}\left(P_{n-2}\right) \rightarrow \operatorname{Irr}\left(\tilde{P}_{n-2}\right), \mu \mapsto \tilde{\mu}$, defined by $\operatorname{Res}_{\tilde{P}_{n-2}^{*}}^{P_{n-2}^{*} \times A}\left(\mu^{*} \times 1_{A}\right)=\tilde{\mu}$, is a bijection.

Since $\lambda$ is linear and $T_{n}$ is the semi-direct product of $U$ with $\tilde{P}_{n-2}$, it follows that $\lambda$ extends to an irreducible character $\hat{\lambda}$ of $T_{n}$ such that $\operatorname{Res}_{\tilde{P}_{n-2}}^{T_{n}}(\hat{\lambda})=1_{\tilde{P}_{n-2}}$. Thus if $\mu$ is an irreducible character of $P_{n-2}$, the induced character

$$
{ }^{2} \psi_{\mu}:=\operatorname{Ind}_{T_{n}}^{P_{n}}\left(\hat{\lambda} \cdot \operatorname{Infl}_{\tilde{P}_{n-2}}^{T_{n}}(\tilde{\mu})\right)
$$

is an irreducible character of $P$ of Type 2. Moreover, every irreducible character of $P$ of Type 2 arises this way. We have ${ }^{2} \psi_{\mu}(1)=\left(q^{n-2}-1\right) \mu(1)$.

2.3.3. Characters of Type 3. The description of these characters depends on the parity of $q$, and we only describe them in the case of odd $q$. Here, the non-trivial irreducible characters of $Z(U)$ fall into two orbits under the action of $A$. Let $\zeta_{1}$ and $\zeta_{2}$ be representatives of the two orbits. There are unique irreducible characters $\rho_{i}$ of $U$ with $\rho_{i}(1)=q^{m-1}$ (recall that $n=2 m$ ) such that $\operatorname{Res}_{Z(U)}^{U}\left(\rho_{i}\right)=q^{m-1} \zeta_{i}$, $i=1,2$.

Since $L^{\prime}=\operatorname{Sp}_{n-2}(q)$ acts trivially on $Z(U)$, the $\rho_{i}$ are invariant under $L^{\prime}$. Trivially, they are also invariant under $Z=Z(G)=\left\langle-I_{n}\right\rangle$, which is contained in $P$ but not in $P^{\prime}$. Since every element of $P$ that fixes $\rho_{i}$ also fixes $\zeta_{i}$, it follows that $P^{\prime} Z=P^{\prime} \times Z$ is the full stabilizer of $\rho_{i}$ in $P, i=1,2$. Now the $\rho_{i}$ extend to characters $\hat{\rho}_{i}$ of $P^{\prime}=U L^{\prime}$ (see [5, Theorem 2.4]). We denote the trivial extensions of the $\hat{\rho}_{i}$ to $P^{\prime} Z$ by the same symbols.

Every irreducible character $\vartheta$ of $L^{\prime} \cong \operatorname{Sp}_{n-2}(q)$ has two extensions to $L^{\prime} Z=$ $L^{\prime} \times Z$, namely $\vartheta \cdot 1_{Z}^{\varepsilon}$ with the sign $\varepsilon \in\{+,-\}$, where $1_{Z}^{+}$and $1_{Z}^{-}$denote the trivial and non-trivial irreducible characters of $Z$, respectively. For each such $\vartheta$ we thus get four irreducible characters of $P$ of Type 3:

$$
{ }^{3} \psi_{\vartheta}^{i, \varepsilon}:=\operatorname{Ind}_{P^{\prime} Z}^{P}\left(\hat{\rho}_{i} \cdot \operatorname{Inf}_{L^{\prime} Z}^{P^{\prime} Z}\left(\vartheta \cdot 1_{Z}^{\varepsilon}\right)\right),
$$

$i=1,2$ and $\varepsilon \in\{+,-\}$. Every character of Type 3 arises this way. We have ${ }^{3} \psi_{\vartheta}^{i, \varepsilon}(1)=q^{m-1}(q-1) \vartheta(1) / 2$.

\section{Restricting the Steinberg character}

3.1. The restriction. For every standard Levi subgroup $M$ of $G$, we denote the Steinberg character of $M$ by $\mathrm{St}_{M}$. We want to compute the restriction $\operatorname{Res}_{P}^{G}\left(\mathrm{St}_{G}\right)$. It is well known that $\operatorname{Res}_{P}^{G}\left(\operatorname{St}_{G}\right)=\operatorname{Ind}_{L}^{P}\left(\operatorname{St}_{L}\right)$ (see e.g., [1, Proposition 6.3.3]); here $L$ is the standard Levi complement $L_{n-2}$ of $P=P_{n}$. We thus have to compute 
$\operatorname{Ind}_{L}^{P}\left(\mathrm{St}_{L}\right)$. We can do this more generally by replacing $\mathrm{St}_{L}$ by irreducible characters of $L^{\prime}=\operatorname{Sp}_{n-2}(q)$, extended trivially to $L=L^{\prime} \times(q-1)$. Notice that $\mathrm{St}_{L}$ is the trivial extension of $\mathrm{St}_{L^{\prime}}$ to $L$.

In computing the multiplicities of characters of Type 3 in such induced characters for odd $q$, the Weil characters of $L^{\prime}$ play a crucial role. Recall that $\hat{\rho}_{i}$ denotes an extension of $\rho_{i}$, an irreducible character of $U_{n-2}$, to $P_{n-2}^{\prime}=U_{n-2} L_{n-2}^{\prime}$. If $q$ is odd and $n \geq 4$, the characters $\omega_{i}:=\operatorname{Res}_{L_{n-2}^{\prime}}^{P_{n-2}^{\prime}}\left(\hat{\rho}_{i}\right), i=1,2$ are called the Weil characters of $L^{\prime} \cong \operatorname{Sp}_{n-2}(q)$. Gérardin has shown in [5, Corollary 4.4], that $\omega_{i}$ is the sum of two irreducible characters of degrees $\left(q^{(n-2) / 2}-1\right) / 2$ and $\left(q^{(n-2) / 2}+1\right) / 2$, respectively.

If $H$ is a finite group, we write $\langle-,-\rangle_{H}$ for the usual inner product on the set of class functions on $H$.

Proposition 3.2. Suppose that $q$ is odd and that $n \geq 4$. Let $\sigma$ be an irreducible character of $L^{\prime}=L_{n-2}^{\prime} \cong \operatorname{Sp}_{n-2}(q)$. Denote the trivial extension of $\sigma$ to $L=L^{\prime} \times A$ by $\sigma$ as well. Then we have

$$
\begin{aligned}
\operatorname{Ind}_{L}^{P}(\sigma) & ={ }^{1} \psi_{\sigma} \\
& +\sum_{\mu \in \operatorname{Irr}\left(P_{n-2}\right)}\left\langle\operatorname{Res}_{P_{n-2}}^{L^{\prime}}(\sigma), \mu\right\rangle_{P_{n-2}}{ }^{2} \psi_{\mu} \\
& +\sum_{\vartheta \in \operatorname{Irr}\left(L^{\prime}\right)}\left\langle\sigma \cdot \omega_{1}, \vartheta\right\rangle_{L^{\prime}}{ }^{3} \psi_{\vartheta}^{1,+}+\left\langle\sigma \cdot \omega_{2}, \vartheta\right\rangle_{L^{\prime}}{ }^{3} \psi_{\vartheta}^{2,+} .
\end{aligned}
$$

Proof. Let us begin with characters of Type 1. Let $\tau \in \operatorname{Irr}(L)$. We have

$$
\begin{aligned}
\left\langle\operatorname{Ind}_{L}^{P}(\sigma),{ }^{1} \psi_{\tau}\right\rangle_{P} & =\left\langle\sigma, \operatorname{Res}_{L}^{P}\left(\operatorname{Infl}_{L}^{P}(\tau)\right)\right\rangle_{L} \\
& =\langle\sigma, \tau\rangle_{L},
\end{aligned}
$$

and thus ${ }^{1} \psi_{\sigma}$ is the only constituent of $\operatorname{Ind}_{L}^{P}(\sigma)$ of Type 1 .

Let us now consider characters of Type 2 , and let $\mu \in \operatorname{Irr}\left(P_{n-2}\right)$. In the following computation we use Mackey's theorem together with the fact that $T_{n} L=P$ and that $L \cap T_{n}=\tilde{P}_{n-2}$. We have

$$
\begin{aligned}
\left\langle\operatorname{Ind}_{L}^{P}(\sigma),{ }^{2} \psi_{\mu}\right\rangle_{P} & =\left\langle\sigma, \operatorname{Res}_{L}^{P}\left({ }^{2} \psi_{\mu}\right)\right\rangle_{L} \\
& =\left\langle\sigma, \operatorname{Res}_{L}^{P}\left(\operatorname{Ind}_{T_{n}}^{P}\left(\hat{\lambda} \cdot \operatorname{Infl}_{\tilde{P}_{n-2}}^{T_{n}}(\tilde{\mu})\right)\right)\right\rangle_{L} \\
& =\left\langle\sigma, \operatorname{Ind}_{L \cap T_{n}}^{L}\left(\operatorname{Res}_{L \cap T_{n}}^{T_{n}}\left(\hat{\lambda} \cdot \operatorname{Inf}_{\tilde{P}_{n-2}}^{T_{n}}(\tilde{\mu})\right)\right)\right\rangle_{L} \\
& \left.=\left\langle\operatorname{Res}_{\tilde{P}_{n-2}}^{L}(\sigma), \operatorname{Res}_{\tilde{P}_{n-2}}^{T_{n}}\left(\hat{\lambda} \cdot \operatorname{Infl}_{\tilde{P}_{n-2}}^{T_{n}}(\tilde{\mu})\right)\right)\right\rangle_{\tilde{P}_{n-2}} \\
& =\left\langle\operatorname{Res}_{\tilde{P}_{n-2}}^{L}(\sigma), \tilde{\mu}\right\rangle_{\tilde{P}_{n-2}} .
\end{aligned}
$$

Recall that $L=L^{\prime} \times A$ and $\sigma=\sigma \times 1_{A}$. Thus

$$
\begin{aligned}
\operatorname{Res}_{P_{n-2} \times A}^{L^{\prime} \times A}\left(\sigma \times 1_{A}\right) & =\operatorname{Res}_{P_{n-2}}^{L^{\prime}}(\sigma) \times 1_{A} \\
& =\sum_{\mu \in \operatorname{Irr}\left(P_{n-2}\right)}\left\langle\operatorname{Res}_{P_{n-2}}^{L^{\prime}}(\sigma), \mu\right\rangle\left(\mu \times 1_{A}\right) .
\end{aligned}
$$

Now $P_{n-2} \times A=\tilde{P}_{n-2} \times A$, and $\operatorname{Res}_{\tilde{P}_{n-2}}^{P_{n-2} \times A}\left(\mu \times 1_{A}\right)=\tilde{\mu}$, which proves the claim for characters of Type 2 . 
Finally, let $\vartheta \in \operatorname{Irr}\left(L_{n-2}^{\prime}\right)$, let $i \in\{1,2\}$ and $\varepsilon \in\{+,-\}$. We shall use the fact that $P^{\prime} Z L=P$ and that $L \cap P^{\prime} Z=L^{\prime} Z$. We have

$$
\begin{aligned}
\left\langle\operatorname{Ind}_{L}^{P}(\sigma),{ }^{3} \psi_{\vartheta}^{i, \varepsilon}\right\rangle_{P} & =\left\langle\sigma, \operatorname{Res}_{L}^{P}\left(\operatorname{Ind}_{P^{\prime} Z}^{P}\left(\hat{\rho}_{i} \cdot \operatorname{Infl}_{L^{\prime} Z}^{P^{\prime} Z}\left(\vartheta \cdot 1_{Z}^{\varepsilon}\right)\right)\right)\right\rangle_{L} \\
& =\left\langle\sigma, \operatorname{Ind}_{L \cap P^{\prime} Z}^{L}\left(\operatorname{Res}_{L \cap P^{\prime} Z}^{P^{\prime} Z}\left(\hat{\rho}_{i} \cdot \operatorname{Inf}_{L^{\prime} Z}^{P^{\prime} Z}\left(\vartheta \cdot 1_{Z}^{\varepsilon}\right)\right)\right)\right\rangle_{L} \\
& =\left\langle\operatorname{Res}_{L^{\prime} Z}^{L}(\sigma), \operatorname{Res}_{L^{\prime} Z}^{P^{\prime} Z}\left(\hat{\rho}_{i}\right) \cdot\left(\vartheta \cdot 1_{Z}^{\varepsilon}\right)\right\rangle_{L^{\prime} \times Z} \\
& =\left\langle\sigma, \operatorname{Res}_{L^{\prime}}^{P^{\prime}}\left(\hat{\rho}_{i}\right) \cdot \vartheta\right\rangle_{L^{\prime}}\left\langle 1_{Z}, 1_{Z}^{\varepsilon}\right\rangle_{Z} \\
& =\left\langle\sigma, \omega_{i} \cdot \vartheta\right\rangle_{L^{\prime}}\left\langle 1_{Z}, 1_{Z}^{\varepsilon}\right\rangle_{Z} \\
& =\left\langle\sigma \cdot \bar{\omega}_{i}, \vartheta\right\rangle_{L^{\prime}}\left\langle 1_{Z}, 1_{Z}^{\varepsilon}\right\rangle_{Z},
\end{aligned}
$$

where $\bar{\omega}_{i}$ denotes the complex conjugate of $\omega_{i}$. So the character ${ }^{3} \psi_{\vartheta}^{i, \varepsilon}$ only occurs in $\operatorname{Ind}_{L}^{P}(\sigma)$ if $\varepsilon=+$, and then it occurs with multiplicity $\left(\sigma \cdot \bar{\omega}_{i}, \vartheta\right)$. By construction of the Weil characters, $\bar{\omega}_{i}$ is again a Weil character, and the result follows.

The above description easily extends to the case of $n=2$. In this case $L=A$ and $L^{\prime}$ is the trivial group. Also, $P / Z$ is a Frobenius group of order $q(q-1) / 2$. Thus $P$ has two irreducible characters of degree $(q-1) / 2$ which have $Z$ in their kernel. These are the characters named ${ }^{3} \psi_{1}^{i,+}, i=1,2$, and $1_{L}^{P}=1_{P}+{ }^{3} \psi_{1}^{1,+}+{ }^{3} \psi_{1}^{2,+}$.

Corollary 3.3. Suppose that $q$ is odd and that $n \geq 4$. Then we have

$$
\begin{aligned}
\operatorname{Res}_{P}^{G}\left(\mathrm{St}_{G}\right) & ={ }^{1} \psi_{\mathrm{St}_{L}}\left\langle\operatorname{Res}_{P_{n-2}}{ }^{L^{\prime}}\left(\operatorname{St}_{L^{\prime}}\right), \mu\right\rangle_{P_{n-2}}{ }^{2} \psi_{\mu} \\
& +\sum_{\mu \in \operatorname{Irr}\left(P_{n-2}\right)}\left\langle\sum_{\vartheta \in \operatorname{Irr}\left(L^{\prime}\right)}\left\langle\operatorname{St}_{L^{\prime}} \cdot \omega_{1}, \vartheta\right\rangle_{L^{\prime}}{ }^{3} \psi_{\vartheta}^{1,+}+\left\langle\operatorname{St}_{L^{\prime}} \cdot \omega_{2}, \vartheta\right\rangle_{L^{\prime}}{ }^{3} \psi_{\vartheta}^{2,+} .\right.
\end{aligned}
$$

This shows in particular that the constituents of Type 2 of $\operatorname{Res}_{P}^{G}\left(\operatorname{St}_{G}\right)$ can be found recursively.

3.4. Constituents of Type 3. To find the multiplicities of the Type 3 characters in the restriction of $\mathrm{St}_{G}$ to $P$, we have to compute the multiplicities of the irreducible constituents of the product $\mathrm{St}_{L^{\prime}} \cdot \omega_{i}$ for $i=1,2$. It follows from the work of Gérardin (see [5, Corollary 4.8.1]) that $\omega_{1}$ and $\omega_{2}$ agree on semisimple elements. On the other hand, the Steinberg character vanishes on elements which are not semisimple. Hence $\mathrm{St}_{L^{\prime}} \cdot \omega_{1}=\mathrm{St}_{L^{\prime}} \cdot \omega_{2}$.

To simplify notation we discuss the analogous product of characters in $G$. Thus let $\mathrm{St}:=\mathrm{St}_{G}$ and let $\omega$ be one of the Weil characters of $G$. The class function St $\cdot \omega$ vanishes except on semisimple elements. Hence it is a linear combination of Deligne-Lusztig generalized characters $R_{T, \vartheta}$, where $T$ runs through the maximal tori of $G$ and $\vartheta$ through the irreducible characters of $T$ (see [1, Corollary 7.5.7]). The Deligne-Lusztig characters $R_{T, \vartheta}$ are either equal or orthogonal (see [3, Corollary 4.5.5]). Hence in order to write $\mathrm{St} \cdot \omega$ as a linear combination of the $R_{T, \vartheta}$, it suffices to compute the scalar products $\left\langle\mathrm{St} \cdot \omega, R_{T, \vartheta}\right\rangle_{G}=\left\langle\omega, \mathrm{St} \cdot R_{T, \vartheta}\right\rangle_{G}$. 
By [1, Proposition 7.5.4], we have St $\cdot \varepsilon_{G} \varepsilon_{T} R_{T, \vartheta}=\operatorname{Ind}_{T}^{G}(\vartheta)$, where $\varepsilon_{G}$ and $\varepsilon_{T}$ are signs as in $[1$, p. 199]. Therefore,

$$
\begin{aligned}
\left\langle\mathrm{St} \cdot \omega, \varepsilon_{G} \varepsilon_{T} R_{T, \vartheta}\right\rangle_{G} & =\left\langle\omega, \mathrm{St} \cdot \varepsilon_{G} \varepsilon_{T} R_{T, \vartheta}\right\rangle_{G} \\
& =\left\langle\omega, \operatorname{Ind}_{T}^{G}(\vartheta)\right\rangle_{G} \\
& =\left\langle\operatorname{Res}_{T}^{G}(\omega), \vartheta\right\rangle_{T} .
\end{aligned}
$$

Through the work of Lusztig, the decomposition of the $R_{T, \vartheta}$ into irreducible characters can be computed. This will be used in the following section to write the tensor product St $\otimes \omega$ as a sum of irreducible characters in the case of $G=\operatorname{Sp}_{4}(q)$.

3.5. An example. To illustrate Corollary 3.3, we compute the restriction of the Steinberg character of $\operatorname{Sp}_{4}(q), q$ odd, to the maximal parabolic subgroup $P_{4}$.

Let $q$ be odd, $G=\operatorname{Sp}_{4}(q), P=P_{4}$, and $L=L_{4}=L^{\prime} \times A$. We have $L^{\prime} \cong$ $\mathrm{Sp}_{2}(q) \cong \mathrm{SL}_{2}(q)$ and its character table can easily be found in the literature (e.g., in $[14$, p. 128]).

(a) We first determine the decomposition of the product of the Weil characters $\omega_{i}, i=1,2$ of $L^{\prime}$ with its Steinberg character St:

$$
\mathrm{St} \cdot \omega_{1}=\mathrm{St} \cdot \omega_{2}=\sum_{\substack{\vartheta \in \operatorname{Irr}\left(\mathrm{SL}_{2}(q)\right) \\ \vartheta(1) \neq 1,(q-1) / 2}} \vartheta .
$$

This can easily be checked with the character table of $\mathrm{SL}_{2}(q)$. The decomposition has been found with the help of CHEVIE [4].

(b) We next determine the restriction of $\mathrm{St}_{L^{\prime}}$ to $P_{2}$. By the remarks following Proposition 3.2 we have $\operatorname{Res}_{\tilde{P}_{2}}^{L^{\prime}}\left(\mathrm{St}_{L^{\prime}}\right)=1+\mu_{1}+\mu_{2}$, where the two characters $\mu_{i}$ have degree $(q-1) / 2$.

(c) From Corollary 3.3 we obtain

$$
\begin{aligned}
\operatorname{Res}_{P}^{G}\left(\operatorname{St}_{G}\right)=\operatorname{Ind}_{L}^{P}\left(\operatorname{St}_{L}\right) & ={ }^{1} \psi_{\mathrm{St}_{L}} \\
& +{ }^{2} \psi_{1}+{ }^{2} \psi_{\mu_{1}}+{ }^{2} \psi_{\mu_{2}} \\
& +\sum_{\substack{\vartheta \in \operatorname{Irr}\left(\mathrm{SL}_{2}(q)\right) \\
\vartheta(1) \neq 1,(q-1) / 2}}{ }^{3} \psi_{\vartheta}^{1,+}+{ }^{3} \psi_{\vartheta}^{2,+} .
\end{aligned}
$$

In particular, $\operatorname{Res}_{P}^{G}\left(\mathrm{St}_{G}\right)$ is multiplicity free.

\section{Decomposition of the Character $\mathrm{St} \cdot \omega \mathrm{IN} \operatorname{Sp}_{4}(q)$}

Let $q$ be an odd prime power and let $\omega$ be one of the two Weil characters of $G=\operatorname{Sp}_{4}(q)$. In this section we will show that the product $\mathrm{St}_{G} \cdot \omega$ is multiplicity-free. By Corollary 3.3 and the result of Subsection 3.5, this implies that for $G=\operatorname{Sp}_{6}(q)$, the restriction of $\mathrm{St}_{G}$ to $P$ is multiplicity free as well.

Let $G=\operatorname{Sp}_{4}(q)$. In order to decompose $\mathrm{St} \cdot \omega$, we shall use the method sketched in Subsection 3.4. The character values of $\omega$ are given explicitly in [15], and our first proof of the following lemma was obtained by a direct computation of the scalar products $\left\langle\operatorname{Res}_{T}^{G}(\omega), \vartheta\right\rangle_{T}$. We are very much indebted to Alex Zalesskii who suggested a more conceptual method for computing these. 
Let $T$ be a maximal torus of $G$, so that

$$
T \in\left\{\mathbb{Z}_{q^{2}+1}, \mathbb{Z}_{q^{2}-1}, \mathbb{Z}_{q-1} \times \mathbb{Z}_{q-1}, \mathbb{Z}_{q+1} \times \mathbb{Z}_{q+1}, \mathbb{Z}_{q+1} \times \mathbb{Z}_{q-1}\right\}
$$

In the notation of $[15], \mathbb{Z}_{q^{2}+1}=\langle\zeta\rangle, \mathbb{Z}_{q^{2}-1}=\langle\theta\rangle, \mathbb{Z}_{q-1}=\langle\gamma\rangle$ and $\mathbb{Z}_{q+1}=\langle\eta\rangle$. Given $x \in\{\zeta, \theta, \gamma, \eta\}$, let $\hat{x}$ be the character such that $\hat{x}(x)=\tilde{x}$, where $\tilde{x} \in \mathbb{C}$ is defined in [15].

Lemma 4.1. Let $T$ be a maximal torus and $\vartheta \in \operatorname{Irr}(T)$. Then the non-zero values of the inner product $\left\langle\operatorname{Res}_{T}^{G}(\omega), \vartheta\right\rangle_{T}$ are as given in Table 1 .

\begin{tabular}{|c|r|r|}
\hline$\left\langle\operatorname{Res}_{T}^{G}(\omega), \vartheta\right\rangle_{T}$ & $(T, \vartheta)$ & Conditions \\
\hline \hline 1 & $\left(\mathbb{Z}_{q^{2}+1}, \hat{\zeta}^{k}\right)$ & $k \neq \frac{q^{2}+1}{2}$ \\
& $\left(\mathbb{Z}_{q^{2}-1}, \hat{\theta}^{k}\right)$ & $k \neq \frac{q^{2}-1}{2}$ \\
& $\left(\mathbb{Z}_{q-1} \times \mathbb{Z}_{q-1}, \hat{\gamma}^{k} \times \hat{\gamma}^{\ell}\right)$ & $k \neq \frac{q-1}{2}$ and $\ell \neq \frac{q-1}{2}$ \\
& $\left(\mathbb{Z}_{q+1} \times \mathbb{Z}_{q+1}, \hat{\eta}^{k} \times \hat{\eta}^{\ell}\right)$ & $k \neq \frac{q+1}{2}$ and $\ell \neq \frac{q+1}{2}$ \\
& $\left(\mathbb{Z}_{q+1} \times \mathbb{Z}_{q-1}, \hat{\eta}^{k} \times \hat{\gamma}^{\ell}\right)$ & $k \neq \frac{q+1}{2}$ and $\ell \neq \frac{q-1}{2}$ \\
\hline 2 & $\left(\mathbb{Z}_{q^{2}-1}, \hat{\theta}^{k}\right)$ & $k=\frac{q^{2}-1}{2}$ \\
& $\left(\mathbb{Z}_{q-1} \times \mathbb{Z}_{q-1}, \hat{\gamma}^{k} \times \hat{\gamma}^{\ell}\right)$ & either $k=\frac{q-1}{2}$ or $\ell=\frac{q-1}{2}$ \\
& $\left(\mathbb{Z}_{q+1} \times \mathbb{Z}_{q-1}, \hat{\eta}^{k} \times \hat{\gamma}^{\ell}\right)$ & $k \neq \frac{q+1}{2}$ and $\ell=\frac{q-1}{2}$ \\
\hline 4 & $\left(\mathbb{Z}_{q-1} \times \mathbb{Z}_{q-1}, \hat{\gamma}^{k} \times \hat{\gamma}^{\ell}\right)$ & $k=\ell=\frac{q-1}{2}$ \\
\hline
\end{tabular}

TABLE 1

Proof. Let us denote the regular character of a finite group $H$ by $\operatorname{reg}_{H}$. If $C$ is a cyclic group of even order, we denote by $\alpha_{C}$ its unique non-trivial irreducible complex character with values in $\{ \pm 1\}$.

The following two facts about the restriction of $\omega$ to the maximal tori of $G$ can be derived from [5, Corollary 4.8.1]. If $T=\mathbb{Z}_{q^{2}+1}$, then $\operatorname{Res}_{T}^{G}(\omega)=\operatorname{reg}_{T}-\alpha_{T}$. If $T=\mathbb{Z}_{q^{2}-1}$, then $\operatorname{Res}_{T}^{G}(\omega)=\operatorname{reg}_{T}+\alpha_{T}$. It follows from [5, Corollary 2.5], that $\operatorname{Res}_{\mathrm{Sp}_{2}(q) \times \operatorname{Sp}_{2}(q)}^{G}(\omega)=\mu \otimes \nu$, where $\operatorname{Sp}_{2}(q) \times \operatorname{Sp}_{2}(q)$ is embedded naturally into $G$ through an orthogonal decomposition of the underlying symplectic space, and where $\mu$ and $\nu$ denote Weil characters of $\operatorname{Sp}_{2}(q)$. These remarks easily give the result of Table 1.

Proposition 4.2. In the notation of [15], let $\Omega$ be the subset of $\operatorname{Irr}(G)$ consisting of the characters listed in Table 2 . Then $\langle\mathrm{St} \cdot \omega, \chi\rangle_{G}=1$ or 0 according as $\chi \in \Omega$ or $\chi \in \operatorname{Irr}(G) \backslash \Omega$.

Proof. This follows from the considerations in Subsection 3.4, Lemma 4.1, and the relations between the irreducible characters and the $R_{T, \vartheta}$ given in [15, Sections $5-7]$.

It is clear that the methods used in this section can be generalized to obtain information about the decomposition of $\mathrm{St}_{G} \cdot \omega$ for symplectic groups $G=\operatorname{Sp}_{n}(q)$ of arbitrary degree $n$.

\section{An application to the Decomposition numbers of $\operatorname{Sp}_{6}(q)$}

Let $\ell$ be a prime number bigger than 3 . In this section we are interested in the $\ell$-modular representations of the groups $\operatorname{Sp}_{6}(q)$ for odd prime powers $q$ prime to $\ell$. 


\begin{tabular}{|c|c|c|c|}
\hline Character & Parameter & Character & Parameter \\
\hline \hline$\chi_{1}(k)$ & $k \in R_{1}$ & $-\chi_{2}(\ell)$ & $\ell \in R_{2}$ \\
\hline$\chi_{3}(i, j)$ & $i, j \in T_{1}, i \neq j$ & $\chi_{4}(i, j)$ & $i, j \in T_{2}, i \neq j$ \\
\hline$-\chi_{5}(k, \ell)$ & $k \in T_{2}, \ell \in T_{1}$ & $\chi_{7}(j)$ & $j \in T_{2}$ \\
\hline$\chi_{9}(i)$ & $i \in T_{1}$ & $-\xi_{1}^{\prime}(j)$ & $j \in T_{2}$ \\
\hline$\xi_{3}^{\prime}(i)$ & $i \in T_{1}$ & $-\xi_{21}(j)$ & $j \in T_{2}$ \\
\hline$-\xi_{22}(j)$ & $j \in T_{2}$ & $\xi_{41}(i)$ & $i \in T_{1}$ \\
\hline$\xi_{42}(i)$ & $i \in T_{1}$ & $\Phi_{7}$ & \\
\hline$\Phi_{8}$ & & $\Phi_{9}$ & \\
\hline$\theta_{1}$ & & $\theta_{2}$ & \\
\hline$\theta_{13}$ & & & \\
\hline
\end{tabular}

TABLE 2

Our main result is a proof of a version of Donovan's conjecture for this class of groups.

Theorem 5.1. Let $\ell>3$ be a prime number and $F$ an algebraically closed field of characteristic $\ell$. Fix a non-negative integer $d$. Then there are only finitely many Morita equivalence classes among the unipotent $\ell$-blocks of defect $d$ of the group algebras $F \operatorname{Sp}_{6}(q)$, where $q$ runs through the odd prime powers not divisible by $\ell$.

Proof. It suffices to consider prime powers $q$ such that $\ell$ divides

$$
\left|\operatorname{Sp}_{6}(q)\right|=q^{9}(q-1)^{3}(q+1)^{3}\left(q^{2}+q+1\right)\left(q^{2}+1\right)\left(q^{2}-q+1\right) .
$$

Since we assume that $q$ is not divisible by $\ell$, and $\ell>3$, it follows that $\ell$ divides exactly one of $q-1, q+1, q^{2}+q+1, q^{2}+1$ or $q^{2}-q+1$. In the latter three cases, the defect groups of the blocks considered are cyclic (see [2]), and therefore there are only finitely many of them of a given defect (up to Morita equivalence).

Since $\ell>3$, it does not divide the order of the Weyl group of $\operatorname{Sp}_{6}(q)$. By a result of Puig [12], the unipotent $\ell$-blocks of the groups $\operatorname{Sp}_{6}(q)$, where $q$ runs through the prime powers with $\ell \mid q-1$, are all Morita equivalent to each other.

We may therefore restrict attention to prime powers $q$ such that $\ell \mid q+1$. Moreover, by [7, Propositions 9.1, 9.2], it suffices to show that the Cartan invariants of such blocks are bounded by a function in $d$. By Brauer reciprocity and the fact that the defect of a block bounds its number of modular irreducible characters, we may instead bound the decomposition numbers in terms of $d$. Since the defect groups of the blocks in question are abelian, it suffices, by [8, Proposition 4.5], to show that the decomposition numbers of the unipotent characters in such a block are bounded by a function in the defect.

Let $q$ be a prime power such that $\ell \mid q+1$. The group $G:=\operatorname{Sp}_{6}(q)$ has two unipotent characters lying in the $\operatorname{Sp}_{4}(q)$-Harish-Chandra-series, labelled by $C_{2}^{\prime}$ and $C_{2}^{\prime \prime}$. The other unipotent characters lie in the principal series and hence are labelled by bi-partitions of 6 . The Steinberg character is labelled by $\left(-, 1^{3}\right)$ (see, e.g., $[1,13.7$, 13.8]). By the results of Fong and Srinivasan [2], $G$ has two unipotent $\ell$-blocks, one of cyclic defect containing the unipotent characters with labels $(21,-)$ and $(-, 21)$, and the principal block. Table 3 is an approximation to the decomposition matrix 
of the unipotent characters of the principal block of $G$. The first column of that table gives the labels of the unipotent characters. The other columns correspond to projective $F G$-modules, whose ordinary characters are denoted by $\Phi_{1}, \ldots, \Phi_{10}$. An entry of Table 3 in a row corresponding to the unipotent character $\chi$ and a column corresponding to the projective character $\Phi$ is the inner product $\langle\chi, \Phi\rangle$. Zeroes are not printed. (This table is an approximation to the decomposition matrix in the sense that we make no statements about the indecomposability of the $\Phi_{i}$.)

Let us shortly describe the origin of these projective characters. Let $P$ denote the parabolic subgroup of $G$ considered in Section 2.2 with Levi subgroup $L \cong$ $\mathrm{Sp}_{4}(q) \times \mathrm{GL}_{1}(q)$. The principal $\ell$-block contains five simple $F L$-modules, and there is one unipotent character of $\ell$-defect 0 . The decomposition matrix of $L$ has been determined by Okuyma and Waki [11]. Harish-Chandra inducing the six projective indecomposable characters lying in unipotent blocks of $L$, we obtain the projective characters $\Phi_{1}, \ldots, \Phi_{4}, \Phi_{6}$, and $\Phi_{7}$. These, and the following Harish-Chandra induced characters have been computed with the CHEVIE share package of GAP3, developed by Geck, Lübeck, and Michel (see [13]).

Let $\Psi_{1}$ denote the character of the projective cover of the trivial $F G$-module. Since $\Phi_{1}$ is obtained by inducing the projective cover of the trivial $F P$-module to $G$, it follows that $\Psi_{1}$ is contained in $\Phi_{1}$ (in the sense that $\Phi_{1}-\Psi_{1}$ is a proper character). Moreover, $\Phi_{1}$ has only unipotent constituents (being contained in the permutation character of $G$ on the Borel subgroup of $G$ ). The permutation character of $G$ on $P$ equals $(3,-)+(2,1)+(21,-)$ (we identify a unipotent character with its label). Since $(21,-)$ is not contained in the principal $\ell$-block of $G$, and since $\ell$ divides the index of $P$ in $G$, it follows that the reduction of $(2,1)$ modulo $\ell$ contains a trivial constituent. This implies that $(2,1)$ is a constituent of $\Psi_{1}$. The Steinberg character $\left(-, 1^{3}\right)$ is also contained in $\Psi_{1}$ (see, e.g., [6]). Using the degrees of its constituents, it is now easy to check that $\Psi_{1}=\Phi_{1}$. (Similarly, $\Phi_{2}$, being Harish-Chandra induced from the defect 0 unipotent character of $L$, has no non-unipotent constituents and is the character of an indecomposable projective $F G$-module. Using the $\ell$-modular representation theory of the Iwahori-Hecke algebra of type $C_{3}$ with parameters -1 , Jürgen Müller has given a more elegant proof of the indecomposability of $\Phi_{1}$ and $\Phi_{2}$.)

The projective character $\Phi_{5}$ is the Harish-Chandra induced Gelfand-Graev character of the Levi-subgroup $\operatorname{Sp}_{2}(q) \times \mathrm{GL}_{2}(q)$, and $\Phi_{10}$ is the Gelfand-Graev character of $G$ (restricted to the principal block). It is easy to see that $\Psi=(21,-)+(-, 21)$ is a projective character in the non-principal unipotent block of $G$. The product $\Psi \cdot(21,-)$ contains the character of the projective cover of the trivial $F G$-module, which was shown above to be equal to $\Phi_{1}$, and $\Phi_{8}=\Psi \cdot(21,-)-\Phi_{1}$. Finally, the product $\Phi_{2} \cdot C_{2}^{\prime}$ yields $\Phi_{9}$. The products of these characters have been computed with the Maple-based CHEVIE system (see [4]), containing in particular the table of unipotent characters of $\operatorname{Sp}_{6}(q)$ computed by Lübeck (see [10]).

Corollary 3.3 and Proposition 4.2 show that the restriction of $\mathrm{St}_{G}$ to $P$ is multiplicity free. By the results of Okuyama and Waki [11] on the decomposition numbers of $\mathrm{Sp}_{4}(q)$, and by Clifford theory, the decomposition numbers in $P$ are bounded in terms of $d$, independently of $q$. This in turn implies that the decomposition numbers occurring in $\mathrm{St}_{G}$ are bounded in terms of $d$, completing the proof. $\diamond$ 


\begin{tabular}{|c|c|c|c|c|c|c|c|c|c|c|}
\hline & $\Phi_{1}$ & $\Phi_{2}$ & $\Phi_{3}$ & $\Phi_{4}$ & $\Phi_{5}$ & $\Phi_{6}$ & $\Phi_{7}$ & $\Phi_{8}$ & $\Phi_{9}$ & $\Phi_{10}$ \\
\hline$(3,-)$ & 1 & & & & & & & & & \\
\hline$(2,1)$ & 1 & 1 & & & & & & & & \\
\hline$(-, 3)$ & 1 & & 1 & & & & & & & \\
\hline$C_{2}^{\prime}$ & & & & 1 & & & & & & \\
\hline$(1,2)$ & 1 & 1 & 1 & & 1 & & & & & \\
\hline$\left(1^{2}, 1\right)$ & 1 & 1 & & & 1 & 1 & & & & \\
\hline$\left(1,1^{2}\right)$ & 1 & 1 & 1 & 2 & 1 & 1 & 1 & & & \\
\hline$\left(1^{3},-\right)$ & 1 & & & & & 1 & & 1 & & \\
\hline $\begin{array}{c}C_{2}^{\prime \prime} \\
\end{array}$ & 1 & & & 1 & 1 & & & & $\begin{array}{c}1 \\
1\end{array}$ & \\
\hline$\left(-, 1^{0}\right)$ & 1 & & 1 & 2 & 1 & 1 & 1 & $(q+1) / 2$ & $(q-1) / 2$ & 1 \\
\hline
\end{tabular}

TABLE 3. An approximation to the decomposition matrix of $\operatorname{Sp}_{6}(q)$

Christoph Köhler has shown that in fact all the entries of Table 3 except $(q \pm$ 1)/2 are true decomposition numbers, but this stronger information is not needed to prove the above theorem. Köhler's results will appear as part of his $\mathrm{PhD}$ thesis [9]. It is also clear that the results of Corollary 3.3 and Proposition 4.2 can be used to improve the known bounds on the two unknown decompositon numbers considerably.

Table 3 is also an approximation to the 3-modular decomposition matrix of $G=\operatorname{Sp}_{6}(q)$ if $3 \mid q+1$. In this case, however, the Sylow 3-subgroup of $G$ is not abelian, and we cannot appeal to [8, Proposition 4.5] to bound all decomposition numbers in the principal 3-block by the decomposition numbers occuring for unipotent characters.

\section{ACKNOWLEDGEMENTS}

We thank Meinolf Geck, Frank Lübeck, and Gunter Malle for clarifying discussions on Deligne-Lusztig theory. We also thank Alex Zalesskii for his hints on the restrictions of the Weil characters to maximal tori. These suggestions led to a substantial simplification of some of our computations.

Most of this work was done during a visit of the second author at the Department of Mathematics of the University of Auckland. He wishes to express his sincere thanks to all the persons of the department for their hospitality, and also to the Marsden Fund of New Zealand who supported his visit via grant \#9144/336828.

\section{REFERENCES}

[1] R.W. CARTER. Finite groups of Lie type: Conjugacy classes and complex characters (Wiley, 1985).

[2] P. Fong and B. SRinivasan. The blocks of finite classical groups. J. reine angew. Math. 396 (1989), 122-191.

[3] M. GECK. An introduction to algebraic geometry and algebraic groups (Oxford University Press, 2003).

[4] M. Geck, G. Hiss, F. Lübeck, G. Malle, and G. Pfeiffer. CheVIE - A system for computing and processing generic character tables. AAECC 7 (1996), 175-210.

[5] P. GÉrardin. Weil representations associated to finite fields. J. Algebra 46 (1977), 54-101.

[6] G. Hiss. The number of trivial composition factors of the Steinberg module. Archiv Math. 54 (1990), 247-251. 
[7] G. Hiss and R. Kessar. Scopes reduction and Morita equivalence classes of blocks in finite classical groups. J. Algebra 230 (2000), 378-423.

[8] G. Hiss And R. Kessar. Scopes reduction and Morita equivalence classes of blocks in finite classical groups II. J. Algebra, to appear.

[9] C. KöHLER. Zerlegungszahlen der Chevalley-Gruppen $F_{4}(q)$. Dissertation, RWTH Aachen University, in preparation.

[10] F. LüBECK. Charaktertafeln für die Gruppen $\operatorname{CSp}_{6}(q)$ mit ungeradem $q$ und $\operatorname{Sp}_{6}(q)$ mit geradem $q$. Dissertation, University of Heidelberg (1993).

[11] T. Okuyama and K. Waki. Decomposition numbers of $S p(4, q)$. J. Algebra 199 (1998), $544-555$.

[12] L. Puig. Algèbres de source de certaines blocs de groupes de Chevalley. In Représentations linéaires des groupes finis, Luminy, 16-21 mai 1988. Astérisque 181-182 (1990), pp. 221236.

[13] M. SchÖNerT ET. AL. GAP - Groups, Algorithms, and Programming - version 3 release 4 patchlevel 4, share package CHEVIE. Lehrstuhl D für Mathematik, RWTH Aachen University, 1997.

[14] I. Schur. Untersuchungen über die Darstellung der endlichen Gruppen durch gebrochene lineare Substitutionen. J. reine angew. Math. 132 (1907), 85-137.

[15] B. SRinivasan. The characters of the finite symplectic group $S p(4, q)$. Trans. Amer. Math. Soc. 131 (1968), 488-525.

J.A.: Department of Mathematics, University of Auckland, Private Bag 92019, AuckLand, New Zealand

G.H.: Lehrstuhl D für Mathematik, RWTH Aachen, Templergraben 64, 52062 Aachen, GERMANY

E-mail address: J.A.: an@math.auckland.ac.nz

E-mail address: G.H.: Gerhard.Hiss@Math.RWTH-Aachen.DE 